\title{
Fortaleza Digital: Criação da infraestrutura de integração de dados em um modelo de Smart City Bottom-Up na Cidade de Fortaleza
}

\author{
José Eurico V. Filho ${ }^{1,2}$, Anderson C. S. Uchôa ${ }^{1}$, Daniel M. Brasil ${ }^{1}$, Ricardo Caratti ${ }^{1}$, \\ Flávio N. Carneiro', Cláudio R. G. de Lima ${ }^{1}$ \\ ${ }^{1}$ CITINOVA - Fundação de Ciência Tecnologia e Informação da Prefeitura de Fortaleza \\ - Fortaleza - CE - Brasil. \\ ${ }^{2}$ Núcleo de Aplicação em Tecnologia da Informação - Universidade de Fortaleza - \\ Fortaleza - CE- Brasil. \\ euricovasconcelos@unifor, fanderson.uchoa, daniel.brasil,
ricardo.caratti, flavio.carneiro, claudio.ricardo\}@fortaleza.ce.gov.br
}

Abstract. The increasing urban concentration has made cities complex social ecosystems with innumerable management challenges. In order to face such challenges, it is necessary that this ecosystem be viewed in a holistic way, understanding the complex and multifactorial nature of the demands, being fundamental the use of quality information for decision making and service delivery. Based on the concept of Smart Cities, within a bottom-up view, the city hall of Fortaleza with the support of the academy, has been developing a project to create a robust data management infrastructure in the city to provide information and digital services (for the management and the population) that help in the improvement of the city and the quality of life of the citizen.

Resumo. A crescente concentração urbana vem tornando as cidades ecossistemas sociais complexos com inúmeros desafios de gestão. Para enfrentar tais desafios, é necessário que esse ecossistema seja visto de forma holística, entendendo a natureza complexa e multifatorial das demandas, sendo basilar o uso da informação de qualidade para a tomada de decisão e a prestação de serviço. Amparados no conceito de Smart Cities, dentro de uma visão bottom-up, a prefeitura de Fortaleza com o apoio da academia, vem desenvolvendo um projeto de criação de uma infraestrutura robusta de gestão dos dados da cidade para prover informações e serviços digitais (para a gestão e para a população) que auxiliem na qualidade de vida do cidadão.

\section{Introdução}

A Fundação de Ciência, Tecnologia e Inovação de Fortaleza - CITINOVA é um órgão da administração indireta do Município de Fortaleza que tem como finalidade estabelecer, promover e executar a Política de Inovação e Desenvolvimento Tecnológico do Município de Fortaleza, por meio da efetiva interação entre a Administração Pública Municipal, Universidades e Centros de Pesquisa. 
A partir de sua criação, a CITINOVA preocupa-se em ter um olhar holístico sobre a cidade e a gestão, coordenando e executando ações que envolvam a energia criativa, o conhecimento científico e o desenvolvimento da tecnologia, traduzindo-os em soluções para melhoria da qualidade de vida, da gestão e equacionamento dos problemas urbanos de Fortaleza. Em outras palavras preocupa-se com a estruturação de uma cidade amparada no conceito de cidades inteligentes (resilientes, digitais, inclusivas, ubíquas) [Albino et al., 2015; Lee et al., 2014; Yovanof \& Hazapis, 2009; Jang \& Suh, 2010]. O grande desafio é olhar para a cidade e para a gestão de forma holística, transcendendo os eixos tradicionais da gestão (e.g. saúde, educação, segurança, infraestrutura) e trabalhando de forma transversal e entendendo que a base para isso é a informação. Com base nessa visão a Fundação propôs seu programa norteador, o Programa Fortaleza +Inteligente.

O programa Fortaleza +Inteligente, ancorado nos princípios de cidades inteligentes, tem o objetivo de melhorar a qualidade de vida na cidade, auxiliando na produção, coleta, integração, interpretação e disponibilização de dados e informações sobre a cidade buscando a melhoria dos serviços prestados pela cidade de Fortaleza. $\mathrm{O}$ programa propõe algumas linhas de ação integrando novas ações e projetos a esforços já existentes e em andamento na gestão e na cidade. São 5 eixos fundamentais:

- Cidade Conectada (pessoas, gestão e coisas): contemplando os projetos de Wi-Fi público e gratuito (Wi-For), o projeto de conexão dos equipamentos da prefeitura em uma rede de fibra óptica de dados de alta velociadade (FibraFor) e a criação da rede de internet das coisas da cidade (IoTNET4);

- Ciência para a Cidade: projeto de criação de espaços para a difusão da ciência e da cultura digital na cidade (Vila da Cultura Digital);

- Cidade inovadora: projeto de estruturação de um ecossistema municipal de inovação (Incubaworking) e o programa de bolsas para projetos de pesquisa aplicada aos problemas da cidade, cedendo a cidade como um living lab (Citilab).

- Fortaleza Digital: Criação da infraestrutura integrada de dados e serviços digitais da cidade;

O foco do presente trabalho se dá sobre o Fortaleza Digital projeto estruturante para uma gestão e prestação de serviços inteligente.

$\mathrm{Na}$ perspectiva de uma cidade digital [Lee et al, 2014, Petrolo et al, 2014, Yovanof et al, 2009], uma primeira iniciativa é a criação de uma infraestrutura de trânsito de dados e informações e digitalização de serviços e sobre essa infraestrutura uma plataforma digital de relacionamento com o cidadão que ofereça múltiplos canais de atendimento e que possibilite integração com os serviços, legados ou não, prestados pela Prefeitura Municipal de Fortaleza (PMF). Espera-se com o Fortaleza Digital, a redução das barreiras encontradas pelo cidadão ao tentar obter algum tipo de serviço e que são causadas, em geral, pela ação humana (servidor ou prestador de serviço terceirizado), transpondo os feudos digitais existentes atualmente. Diante disso, como outros objetivos importantes do Fortaleza Digital, temos a redução do número de interações humanas na execução dos serviços online, melhorando dessa forma, a lisura dos processos e, consequentemente, a confiança do cidadão e a criação de uma estrutura 
de integração de dados digitais aqui denominada de Barramento de Dados e Serviços. É importante ressaltar que a concepção original do Fortaleza Digital tem como meta a ausência de interação humana nos serviços online publicados na plataforma. Assim, urge a necessidade de constante atualização, modernização e integração, uma vez que nela são desenvolvidos projetos tecnológicos de atualização e desenvolvimento desta Fundação no que compete a sua função.

Com uma abordagem Bottom-Up o Fortaleza Digital visa primeiramente organizar e integrar a infraestrutura de dados e serviços para prover com a máxima velocidade, qualidade e confiabilidade os serviços para o cidadão, de tal maneira a estruturar os dados para prover inteligência para sistemas e tomadas de decisões quanto a cidade.

A interação dos sistemas de uma cidade pode ser considerada inteligente quando faz uso de forma estratégica de suas informações e comunicações para uma melhor resposta à população sobre questões de gestão da cidade. Sendo assim o Fortaleza Digital será um dos pilares centrais para tornar o município de Fortaleza-CE uma cidade mais inteligente.

\section{Cidades Inteligentes}

São vários os conceitos que buscam significar as cidades atualmente. Cada conceito utilizado é uma tentativa de demonstrar ao mundo o quão avançada uma cidade é, seja tecnologicamente ou no campo da inovação. Porém, muitos desses conceitos se confundem e se sobrepõem uns aos outros, sendo a semelhança principal entre todos a consciência que, para qualquer projeto desses funcionar, este deve ser orientado ao cidadão. É importante lembrar que uma cidade é um grande sistema complexo formado por outros sistemas, sejam estes de serviços de infraestrutura, de rede elétrica, de materiais, de pessoas, e etc., que buscam interagir entre si da melhor forma objetivando uma melhor qualidade de vida [Bettencourt, 2015].

A interação dos sistemas de uma cidade pode ser considerada inteligente quando faz uso de forma estratégica de suas informações e comunicações para uma melhor resposta à população sobre questões de gestão da cidade. Sendo assim, a Cidade Inteligente pode ser definida como uma cidade que utiliza a tecnologia da informação e comunicação para melhorar a qualidade de vida da população, estando os dados obtidos da cidade no centro desse processo. As cidades inteligentes também podem ser definidas como territórios que trazem sistemas de inovação e instituições de ciência e tecnologia (ICTs) dentro da mesma localidade, combinando a criatividade de indivíduos talentosos que compõem a população da cidade, instituições que melhoram a aprendizagem e espaços de inovação, geralmente virtuais, que facilitam a gestão do conhecimento. A combinação de criatividade das pessoas envolve a estratégia de inteligência coletiva, onde as tendências são identificadas e padronizadas, utilizando as experiências das pessoas de forma a colaborar coletivamente. Esse conceito também é pautado no uso de ICTs visando a tornar os componentes de infraestrutura e serviços essenciais de uma cidade mais inteligente, interligado e eficiente.

A caracterização de uma cidade inteligente, ou smart city, parte do ponto de vista de que a tecnologia é fator indispensável para que as cidades possam se modernizar, oferecendo melhor infraestrutura e condições de vida a população. Esse 
conceito tem se mostrado fundamental na transformação dos centros urbanos em espaços mais eficientes, além de oferecer boa qualidade de vida e gestão de recursos naturais por meio de um processo participativo.

As cidades são consideradas inteligentes quando são identificadas contendo investimentos inteligentes ao longo dos eixos: economia, mobilidade, meio ambiente, recursos humanos e estilos de vida inteligentes.

$\mathrm{O}$ uso adequado da internet traz o cidadão para junto do governo, facilitando o relacionamento entre ambos. Um exemplo desta aproximação é que, através do fornecimento de informações aos cidadãos, os mesmos podem resolver suas solicitações sem a necessidade de estar presente em um ponto físico de atendimento, melhorando a qualidade no atendimento, reduzindo filas e proporcionando maior agilidade na prestação do serviço público [Chourabi et al., 2012].

De acordo com Komninos et. al. (2011), as tecnologias para o desenvolvimento das cidades são aquelas que fazem uso de sistemas e organizações para ajudar a lidar com as tensões provocadas pelo aumento massivo da urbanização e que através de soluções de colaboração tiraram proveito da diversidade, produzindo cidades eficientes e agradáveis para se viver e trabalhar.

Essa crescente urbanização informatizada traz mais qualidade ao acompanhamento de sistemas em tempo real, bem como a sua administração e melhoria. Porém o conceito de smart cities não está ligado somente à tecnologia, mas também à gestão, governança, infraestrutura e principalmente capital humano e social que em conjunto vislumbram o desenvolvimento econômico e sustentável das cidades.

\section{Infraestrutura de Integração de Dados e Serviços de Fortaleza}

Existem diversos conceitos para uma cidade inteligente, dentre eles podemos citar Bollier (1998) apud Harrison e Donnelly (2011), definindo que o conceito de cidade inteligente teve início com o movimento conhecido por crescimento inteligente, nos anos 1990, que defendia a criação e implantação de políticas urbanas inovadoras. Desde então, o conceito evoluiu para, atualmente, significar formas de gestão urbana alicerçadas em Tecnologia da Informação e Comunicação (TIC).

"Os significativos avanços tecnológicos e das tecnologias da informação e comunicação (TIC) agora fazem das plataformas tecnológicas embarcadas um instrumento potencialmente significativo para sensorizar e monitorar a funcionalidade e o desempenho das cidades, permitindo ampliar sobremaneira suas capacidades de gerenciar recursos com mais eficiência e prover conectividade e informações de forma transparente aos seus cidadãos e visitantes" [C40, 2011, p. 32].

Desta maneira, para tornar uma cidade mais inteligente é necessário ter dados para análise histórica e predição futura, tão importante quanto a disponibilização e integração dos dados é a qualidade com que os mesmos são disponibilizados.

O Fortaleza Digital deve atuar em duas grandes frentes, camada de atendimento ao cidadão e camada de integração de dados e serviços digitais onde: 
Para a camada de atendimento a principal característica é oferecer um ponto único de entrada para o consumo dos serviços prestados pela Prefeitura Municipal de Fortaleza. Consiste de uma interface única para o usuário final. Esta camada deve proporcionar um ambiente que facilite o desenvolvimento, a publicação e a execução dos serviços prestados pela PMF. Este ambiente deve também prover facilidades para que o cidadão possa consultar, resolver problemas e interagir com os serviços prestados pela PMF com o mínimo de interação humana. Esta camada deve oferecer ainda, a possibilidade de implantação de serviços usando outros canais de atendimento diferente da WEB. Por exemplo: Redes Sociais, Chatbots, Dispositivos Móveis (SmartPhones e Tablets). Possibilitando assim o desenvolvimento de novos sistemas e integração de sistemas já existentes.

Para a camada de integração de dados e serviços digitais, a qual denominamos de Barramento de Dados, consiste de um ambiente capaz de integrar várias bases de dados distribuídas em diferente unidades físicas da PMF e por diferentes fornecedores de Sistema de Gerenciamento de Banco de Dados. Essa integração tem como meta oferecer uma visão única dos dados armazenados pelos vários serviços providos pela PMF, facilitando dessa forma, a implementação de novos serviços, o controle do acesso à informação e estudos envolvendo dados de várias secretarias. Pretende-se também com essa camada, melhorar a qualidade dos dados e, consequentemente, a confiabilidade da informação, transpondo as barreiras de feudos de dados, onde existe o conflito quanto a disponibilização dos dados para demais órgãos e secretarias. Para tanto, espera-se desta camada, funções que contribuam também nas atividades relacionadas à qualidade de dados.

É importante ressaltar que o objetivo desta camada não é unificar os bancos de dados dos diversos sistemas digitais providos pelos órgãos e secretarias do município, mas prover o mapeamento e gestão destes dados de forma distribuída.

No que tange à segurança da informação, vale salientar que a PMF já utiliza o modelo de nuvem privada onde todos os sistemas corporativos estão hospedados. Cada sistema implantado neste modelo em nuvem segue critérios rigorosos de segurança da informação. Neste contexto, a plataforma do Fortaleza Digital será mais um sistema inserido neste modelo a seguir as políticas de segurança estabelecidas pela Prefeitura Municipal de Fortaleza.

Desta maneira o Barramento de Dados se torna uma importante ferramenta na concepção de uma infraestrutura de sistemas e dados para tornar uma cidade mais inteligente. Integrando e possibilitando que os dados sejam utilizados não somente de forma vertical, na própria secretaria ou órgão, mas de forma horizontal, podendo estes dados serem disponibilizados para outros órgãos e secretarias, além dos próprios cidadãos.

\section{Arquitetura do Barramento de Dados}

Um grande problema identificado na PMF é a desintegração, replicação e desatualização de dados. Encontrou-se durante a fase de análise da atual estrutura de dados e serviços uma informação específica, sendo provida por diferentes fontes (secretarias ou serviços internos), em diferentes formatos e com diferentes conteúdos 
(desatualizados ou errados). Desta maneira é impossível responder à pergunta de quais serviços o cidadão utilizou e se o mesmo teve seu problema resolvido de forma efetiva.

O esforço e complexidade para unificar as bases de dados dos diversos sistemas de secretarias e órgãos da PMF é impraticável tendo em vista a necessidade de continuidade do serviço. Por mais que os dados sejam corporativos e únicos existem diversas barreiras a serem transpostas. Pensando nisto foi proposta uma arquitetura de barramento de dados onde os dados poderão permanecer dentro de seus respectivos negócios criando dois conceitos importantes: Gestor de Dados e Cliente de Dados, onde:

Podemos conceituar Gestor de Dados o órgão responsável por gerir os dados de um determinado contexto de negócio, sendo responsável por: Definir regras de gestão deste dado (Acesso e Atualização), prover mecanismos para disponibilização do dado ao barramento e definir e implementar as regras de negócio pertinentes ao dado.

Já o Cliente de Dados serão todos os órgãos e sistemas que farão uso de dados geridos por outros órgãos, o qual poderá: Consumir o dado, gerar uma extensão do dado para atender às suas demandas de negócio, realizar alterações nos dados mediante a permissão do gestor do dado.

A arquitetura proposta utiliza técnicas de virtualização de dados capaz de oferecer um ambiente único para acesso aos repositórios de vários sistemas corporativos. $\mathrm{O}$ acesso aos sistemas continuam seguindo os critérios de segurança corrente. Isto é, conforme sugerido anteriormente, não é proposta deste arquitetura modificar as políticas de segurança em vigor. No entanto, pretende-se com esta técnica, minimizar a redundância de dados existentes em várias bases da PMF.

Para demonstrar uma utilização prática da técnica de virtualização de dados, tem-se o Cadastro de Cidadãos mantido atualmente pela Secretaria de Finanças do Município de Fortaleza (SEFIN), que tem como uma das suas funções institucionais, manter esse cadastro atualizado. Porém, vários sistemas da PMF possuem também cadastro próprios de cidadãos que normalmente não têm a mesma dinâmica de atualização do cadastro da SEFIN, portanto menos confiáveis. Diante disso, a arquitetura proposta tem como uma de suas estratégias, facilitar a substituição de bases de dados menos confiáveis por bases de dados mais confiáveis utilizadas por sistemas legados, contribuindo, dessa forma, com a diminuição da redundância de dados.

Em conjunto com a técnica de virtualização de dados, tem-se o barramento de serviços. Este barramento busca oferecer uma visão única dos serviços expostos como Web Services (SOAP ou Rest) já implementados na prefeitura. Pretende-se com isso, a redução de esforços para o desenvolvimento de novos sistemas bem como a manutenção de sistemas legados. É importante ressaltar que o barramento de dados e serviços tem como pilar uma documentação rica e disponível para os vários atores da PMF.

Para implementação do barramento de dados e serviços citados anteriormente, o mercado já possui algumas soluções que estão aderentes às demandas do Fortaleza Digital. A Red Hat, por exemplo, possui o Red Hat Data Virtualization que implementa as principais técnicas de virtualização de dados e o Red Hat Jboss Fuse, uma plataforma voltada para integração serviços, microsserviços e componentes de aplicativos. 
Com o Barramento de Dados (Figura 1), pode-se observar que o mesmo deve funcionar como um motor de integração de dados e API's para prover serviços digitais de forma atualizada e integrada, possibilitando o reuso de tecnologia e proporcionando uma maior qualidade de dados a ser utilizado em uma Camada de Análise de Dados, a qual tem o propósito de prover informações para a tomada de decisão quanto aos serviços prestados;

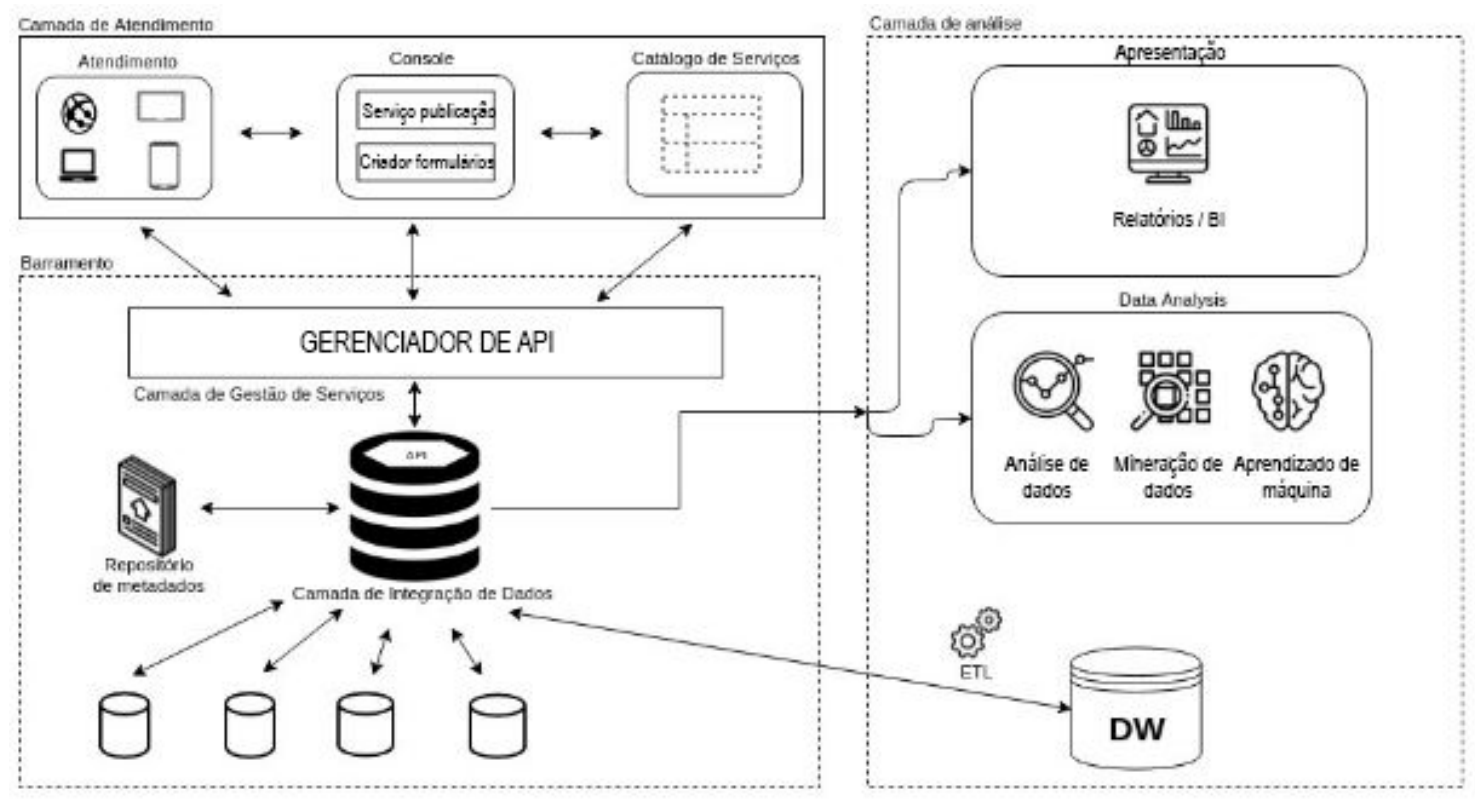

Figura 1. Arquitetura do Fortaleza Digital

Ainda em relação à figura anterior, a camada de atendimento é compreendida pelos componentes responsáveis pela apresentação dos serviços aos usuários finais (cidadãos), identificada na figura por Atendimento. Além do Atendimento, há também os componentes destinados à gestão dos módulos visuais e serviços que serão publicados no Fortaleza Digital, identificados na figura por Console e "Catálogo Serviços". Abaixo da camada de atendimento, há o Barramento de Dados que será composto por duas camadas: Integração de dados e Gestão de API'S.

A camada de integração de dados tem como objetivo gerar uma relação entre os dados que estão distribuídos de forma desagregada em cada contexto de negócio. Como estratégia para atingir este objetivo, será utilizado um componente de virtualização de dados. Este componente irá prover uma camada de abstração para acesso rápido e direto aos dados que estarão distribuídos em bases de dados e SGBDs distintos, e oferecer uma visão única dos dados agregados de forma virtual. A agregação dos dados será possível por meio de um identificador que estará presente nas bases de dados desagregadas, podendo fazer junção de tabelas que podem estar fisicamente em bancos de dados diferentes, baseados neste identificador.

Deste modo, os dados virtualizados poderão ser consumidos por meio de conexão ODBC/JDBC a este componente ou através do consumo de APIs. Aplicando esta estratégia, os dados vão continuar distribuídos e sendo geridos pelo seu respectivo 
contexto de negócio e será possível obter uma visão unificada destes dados virtualmente agregados.

Ainda na camada de integração de dados, será utilizado outro componente para agregar dados provenientes de fontes diversas, como webservices e arquivos, e em formatos diversos. Com este será possível fazer tratamentos, transformações e aplicação de regras de negócio nos dados, que serão expostos via API, utilizando a arquitetura de micro serviços. Desta forma, cada serviço exposto estará tratando de um contexto específico de negócio ou funcionalidade, com isso os serviços estarão fracamente acoplados e com mais independência para serem geridos. Com este componente será possível executar fluxos de sistema que passam por regras de vários contextos de negócio distintos, gerando assim um resultado integrado.

Os dados integrados serão expostos via APIs, e assim será necessário um componente para fazer a gestão destas. Com isso, compomos a última camada do barramento de dados. Este componente permite a criação, análise, proteção e gestão de APIs de forma segura e escalável. As APIs geradas serão conectadas ao Gestor de APIs e este irá expor de forma centralizada os endpoints para consumo. Será possível fazer monitoramento e gestão dos acessos, controle de tráfego, documentação e, em um ambiente único, ter todo o mapeamento dos dados e serviços que estão integrados ou disponíveis para integração. Os desenvolvedores que farão uso dos dados integrados terão acesso a um ambiente que oferecerá o mapeamento das APIs disponíveis, bem como a respectiva documentação, e ainda poderão realizar testes de consumo dos endpoints.

Podemos ainda observar a Camada de Análise de Dados a qual será responsável por prover informações para sistema de apoio a decisão além de disponibilizar dados não sensíveis para os serviços como Portal da Transparência do Município e Dados Aberto.

\section{Conclusão}

O projeto Fortaleza Digital é um projeto que integra gestão pública e academia buscando oferecer uma visão horizontal dos dados e serviços, melhorando o acesso do cidadão ao tais serviços oferecidos pela PMF. Com as ações propostas espera-se a redução dos custos operacionais da PMF na prestação dos serviços, a garantia da segurança e disponibilidade da informação, a privacidade dos usuários (cidadãos), além da facilidade na implementação e implantação de novos serviços oferecidos pela PMF. O projeto ainda em andamento está em fase de implantação e seus resultados serão colhidos a longo prazo. Mas um resultado já de grande impacto foi a integração das diversas áreas de TIC da PMF em uma visão unificada e integrada, bem como a aproximação junto a academia, trazendo vanguarda tecnológica para a PMF. 


\section{References}

Albino, Vito, Umberto Berardi, and Rosa Maria Dangelico, (2015). Smart cities: Definitions, dimensions, and performance. Journal of Urban Technology, volume 22 , number 1 .

Bettencourt, Luís. M. A. (20015). Cidades como Sistemas Complexos. In: Furtado, Bernardo et al. Modelagem de Sistemas Complexos para Políticas Públicas. Brasília: IPEA, 2015. p. 241-263

Bollier, D. (1998). How smart growth can stop sprawl: a fledgling citizen movement expands. Essential Books. 1998.

C40. (2011). São Paulo Climate Summit. São Paulo: Prefeitura de São Paulo, 2011.

Chourabi, H. et al. (2012) Understanding smart cities: an integrative framework. In: Hawaii International Conference on System Sciences, 45th. p. 2289-2297.

Jang, M.; Suh, Soon-Tak (2010). U-City: New Trends of Urban Planning in Korea Based on Pervasive and Ubiquitous Geotechnology and Geoinformation. Springer, Berlin, Heidelberg. Computational Science and Its Applications - ICCSA.

Komninos, Nicos, (2006). The architecture of intelligent cities. Intelligent Environments, Institution of Engineering and Technology, July 2006.

Lee, Jung Hoon; Hancock, Marguerite Gong; Hu, Mei-Chih (2014). Towards an effective framework for building smart cities: Lessons from Seoul and San Francisco. Technological Forecasting and Social Change. doi:10.1016/j.techfore.2013.08.033

Petrolo, R.; Loscrí, V.; Mitton, N. (2014). Towards a Smart City Based on Cloud of Things. New York, NY, USA: ACM. Proceedings of the 2014 ACM International Workshop on Wireless and Mobile Technologies for Smart Cities.

Yovanof, Gregory S.; Hazapis, George N. (2009). An Architectural Framework and Enabling Wireless Technologies for Digital Cities \& Intelligent Urban Environments Wireless Personal Communications. 49 (3): doi:10.1007/s11277-009-9693-4 